\title{
Surface photometry of cD envelopes
}

\author{
S. N. Kemp, V. Guzmán Jiménez, P. Ramírez Beraud, \\ F. J. Hernández Ibarra, and J. A. Pérez Grana
}

Instituto de Astronomía y Meteorología, Universidad de Guadalajara, Av. Vallarta 2602, Col. Arcos Vallarta, CP 44130, Guadalajara, Jalisco, México; email:snk@astro.iam.udg.mx

We have carried out deep $B V R$ surface photometry of $6 \mathrm{cD}$ and $\mathrm{cD}$-like galaxies using the 2.1-m telescope at San Pedro Mártir, $\mathrm{cD}$ galaxies are supergiant galaxies $(M>$ $10^{13} M_{\odot}$ ) with enormous halos (> $100 \mathrm{kpc}$ in radius) surrounding a giant elliptical galaxy, found generally at the centre of rich clusters (Oemler 1976, Schombert 1988). The surface brightness profiles of their halos (envelopes) break from the $r^{1 / 4}$ law, containing more light at large radii (Schombert (1988)), although a detailed 1 and 2 dimensional analysis of their morphology has yet to be carried out. There have been four main theories as to the origin of $\mathrm{cD}$ envelopes (Schombert (1988) and references within), a) stripping of stars from other cluster member galaxies, b) formation of galaxy and envelope at the same time during the formation of the cluster, c) mergers of cluster members, which do not easily explain the high velocity dispersions in the envelopes $\left.\left(\sim 1000 \mathrm{~km} \mathrm{~s}^{-1}\right), \mathrm{d}\right)$ cooling flows: accumulation of cooling X-ray-emitting ICM gas around the central galaxy. Very red envelopes have been found around some cD's, and star formation biased towards lowmass stars in cooling flows were invoked to explain these red halos, but the expected very bright near-IR halos were not detected (Joy et al. 1995 and references within). Previous detailed studies of $\mathrm{cD}$ galaxies (e.g. Mackie 1992) found a range of colour gradients.

Our sample included the cDs in the clusters A193, A426 (NGC 1275), A496, A754, A2634 and A2670. The typical colours of their envelopes are $(B-V)=1.1-1.3$ and $(V-R)=0.5-0.7$. In most cases there is no obvious gradient over the entire radial range studied. In the two cD-like galaxies (A426, A754), a gradient of 0.5 to the red in $(B-R)$ was detected, although no extended envelope was detected (neighbour galaxies make it difficult to follow the profile). For NGC 1275, the central regions are bluer due to many regions of active star formation. In the case of 2 objects with multiple nuclei (A193 and A2634), no colour gradients, nor zones of star formation, were found. In many cases the colours of nearby cluster members are very similar to the $\mathrm{cD}$ galaxy.

These results favour a primordial origin for the $\mathrm{cD}$ envelope. Thus the envelope retains characteristics of the cluster, and its stellar population has evolved similarly to that of the central galaxy. Any changes caused by mergers, interactions, and cooling flows, are temporary. The envelope is dominated by an old stellar population ('red-and-dead'). The largest galaxies in the universe may well be the first to be fully formed, and this seems to be in agreement with recent ideas of 'downsizing' in which $\mathrm{E} / \mathrm{cD}$ galaxies are the first to complete their star formation.

\section{References}

Joy, M., Prestwich, A.H., Burton, M. \& Guimond, S. 1995, ApJ 449, L113.

Mackie, G. 1992, ApJ 400, 65.

Oemler, A., Jr. 1976, ApJ 209, 693.

Schombert, J.M. 1988, ApJ 328, 475. 